\title{
Metacognitive Self-Regulation: The Mediating Role of Emotion Oriented Coping between Positive and Negative Beliefs about Rumination amongst Pakistani Adults
}

\author{
Aisha Muneer, Jamil A Malik, Raiha Aftab
}

\begin{abstract}
OBJECTIVES: The study explored the moderated mediating role of gender on the relationship between positive and negative beliefs about rumination mediated by emotion oriented coping.

METHODOLOGY: The research was a short survey. Data was collected from various institutes of Islamabad and Rawalpindi during 2015-2016. A total of 514 employed professionals from different organizations participated in the study. The questionnaires method was used. Responses were collected on Positive Beliefs about Rumination Scale, Negative Beliefs about Rumination Scales and Emotion Oriented Coping. Correlation analysis showed significant positive relationships among study variables (ranging from .32 to $.40 ; p<.01$ ).

RESULTS: Results showed that direct path between negative beliefs about rumination and positive beliefs about rumination was found mediated by emotion oriented coping. The direct effects between positive beliefs about rumination and negative beliefs about rumination $\left(\beta=.31, p<.01, \Delta R^{2}=.23\right)$, and the indirect effects between positive and negative beliefs about rumination through emotion- oriented coping $\left(\beta=.10, p<.05, \Delta R^{2}=.18\right)$ disclosing mediating effects of emotion oriented coping.

CONCLUSION: Emotional coping was thus found to be a process through which individuals can 'let go of' factors that may be causing them stress and they can attain a temporary sense of calm. Previous research had hinted upon the cathartic nature of emotional coping but it wasn't clear whether the relationship would be significant or not. The current research is step towards providing empirical evidence for this relationship. Secondly, the article specifies the direction of the relationship between positive beliefs about rumination, emotion oriented coping and negative beliefs about rumination.
\end{abstract}

KEYWORDS: Mediation; Positive beliefs about rumination; Emotion oriented coping, Direct effects and Indirect effects.

This article may be cited as: Muneer A, Malik JA, Aftab R. Metacognitive Self-Regulation: The Mediating Role of Emotion Oriented Coping between Positive and Negative Beliefs about Rumination amongst Pakistani Adults. J Liaquat Uni Med Health Sci. 2019;18 (01):71-6. doi: 10.22442/jlumhs.191810604

\section{INTRODUCTION}

The Self-Regulatory Executive Function (S-REF) model conceptualizes rumination as a comprehensive cognitive mechanism that engages automatic processing, in terms of self- knowledge or beliefs we carry about our thoughts and voluntary conscious processing in form of choice of coping techniques. Wells and Mathews ${ }^{1}$ suggest that beliefs, especially in emotionally vulnerable individuals may affect the choice of coping strategy; it may further fixate them into a thinking style that is repetitive, persistent and active about the causes and consequences of rumination triggering events. Here, the role of metacognitions (MC) is of particular importance. Metacognitions (MC) defined as the knowledge of the cognitive processes ${ }^{2}$ play a vital role in forming beliefs, monitoring and allocating self-regulatory strategies.
Rumination is a way of coping that is employed in the presence of, a lack of action- oriented problem solving behavior $^{3}$. It is typically guided by an emotion focused approach. The mechanism of rumination in S-REF model is grounded on a comprehensive architectural design consisting of both automated and voluntary processing ${ }^{4}$. Initially any event or triggering factor is automatically acknowledged by lower level networks for example, sensory mechanisms. Stimuli holding motivationally significant valence are then appraised by involving self-knowledge ${ }^{5}$ and metacognitions for re - evaluating in the light of previous experiences and beliefs that are carried for the situation at hand. This re-evaluation is passed as a feedback to the supervisory executive systems that are responsible for making a decision on choosing an appropriate coping strategy. In emotionally vulnerable individuals coping is manifested in terms of ruminative style for self-regulation that interferes with problem solving ${ }^{3}$ 
resulting into brooding for why something happened and what were the aftermaths. The repetitiveness of these thoughts further aggravates the situation by eroding social relationships as people get tired of listening to the same emotional content.

This design is also suggestive of a universal quality of rumination i.e., rumination occurs in both normal or healthy people and clinical patients. In normal individual this is usually observed into practice whenever there is a goal discrepancy ${ }^{6,7}$. Upon activation of the S-REF the executive mental functions start processing within a narrowed frame with attentional bias, ignoring the neutral, relevant information that contributes to the richness of the experience. This condition is referred to cognitive attentional syndrome (CAS), where multiple executive mental functions such as memory, attention and thinking etc. may be biased effecting the self-knowledge creating atypical perceptual tendencies. The atypical perceptions provide feedback to the supervisory executive system hence impacting the choice for coping strategy. The repetition of thoughts is attributed to the $\mathrm{CAS}^{8}$ that prohibits the attention allocation to neutral information. Emotion oriented coping and lack of problem solving approach is anarchetypal feature of rumination, thus under these provisions a resort for coping would be in terms of emotional orientation. According to Wells A $2008^{9}$ emotion oriented approach of a problematic situation in itself is a source of pathology. Individuals frequently using emotion and avoidance oriented coping can be assumed to be carrying pathological vulnerabilities. Thus it is absolutely necessary to investigate cognitive tendencies leading to choosing emotions as a coping mechanism. In the light of literature we argue that, behavior execution is guided by a belief system ${ }^{10}$, similar to this is the S-REF processing in which the self-knowledge constitutes our cognitions and metacognitions. The metacognitions for a behavior carries positive or negative beliefs that highlight the usefulness or dangers associated with that behavior, hence the choice of strategy in dealing with a situation at hand is most likely to be dependent on the beliefs one holds for those behaviors. Consequently, these positive beliefs are responsible for the choice of an emotion focused style ${ }^{11}$ which contributes to perpetuating ruminative cycle. We sought to investigate if these positive beliefs are responsible for an emotion oriented strategies and serve as a precursor to negative beliefs about rumination. As stated by Wells $\mathrm{A} 2008^{9}$ the emotion and avoidance focused coping may reflect source of pathological vulnerabilities, providing the indication that cognitions lead to such a choice of coping can be assumed to lie in similar directions depicting some usefulness of this choice.

\section{METHODOLOGY}

The research was a short survey. Data was collected from various institutes of Islamabad and Rawalpindi during 2015-2016. A total of 514 participants were selected on convenient sampling technique with a minimum educational level of graduation, employed- working within different faculties in various organizations of Rawalpindi and Islamabad. The age ranged between $25-45$ years. The sample comprised of females $=24 \%$ and males $=76 \%$ from around the twin cities of Pakistan i.e., Rawalpindi and Islamabad. The mean age of the sample was 22.4 (SD=2.6, range 1829).The participants were briefed about the purpose of the present study and were ascertained of the confidentiality of the information provided. The average time taken to fill the questionnaire was 10-minutes approximately. None of the participants found any item complicated or ambiguous.

Positive and Negative Beliefs about Rumination Scale (PBRS \& NBRS): Papageorgiou C and Wells A $2001^{12}$ developed the above scale to assess metacognitive beliefs about rumination. The scale consisted of two subscales: a 9-item subscale that measures positive metacognitive beliefs concentrated on the advantages of the ruminative thinking pattern e.g., "Ruminating about the past helps me to prevent future mistakes and failures". The second subscale consisted of 13-items that focused the danger and uncontrollability of the ruminative thoughts. For both subscales the respondents rate each statement to the extent they approve of each item by using a 4-point Likert scale extending between Do not agree and Agree very much, with high scores representing the strong positive or negative metacognitive beliefs about rumination. The original internal consistency and test-retest reliability coefficients were reported as 0.89 and 0.85 , for PBRS and NBRS respectively. The positive correlations between PBRS and rumination $(r=0.53)$, and PBRS and depressive symptoms $(r=$ $0.45)$ indicate the convergent validity of the scale ${ }^{12}$.

Coping Inventory for Stressful Situation (CISS): Endler $\mathrm{N}$ and Parker $\mathrm{J} 1990^{13}$ developed a 48-items questionnaire on coping styles for stressful situations. The questionnaire consisted of three subscales namely, task oriented, emotion oriented and avoidance oriented coping styles consisting of 16-items each. CISS assesses choice of coping strategies in stressful or challenging situations. Respondents rate each statement to the extent they approve of each item by using a 4-point Likert scale extending between Do not agree and Agree very much, with high scores representing the strength of the type or style of coping one frequently employs. 
The original internal consistency and test-retest reliability coefficients were reported between 0.89 and 0.85 , respectively. For the present research we have considered emotion oriented subscale to fulfill the purpose of the study.

The present study investigated the relationship between positive and negative beliefs about rumination mediated by emotion oriented coping. The beliefs serve as implicit planning, guides the self-regulation process therefore, positive beliefs serve to initiate ruminative processing and emotion oriented coping results into dysfunctional vulnerability reflected in terms of negative beliefs. To the best of our knowledge till date, a scarcity of research inquiry had independently explored this relationship despite literature suggesting their close association in the S-REF processing. We maintain that findings from the present study will provide valuable conclusions in understanding precursors to dysfunctional cognitive processing.

\section{RESULTS}

The data was collected using positive beliefs about rumination scale, emotion oriented coping, and negative beliefs about rumination to achieve the study objectives. The demographics show that present study included 514 professionals (males $=76 \%$; females $=24 \%$ ) from different fields of professions with a mean age of 32.56 years. Of the entire sample approximately $33 \%$ were unmarried, $65 \%$ married. On the professional front, the data indicated a mean experience of approximately 9 years and drawing a salary of Rs. 48,310 on the average. The results are presented in Table I. The reliability coefficients show the psychometric appropriateness of the scales used, results in Table II shows the appropriate magnitudes of internal consistency.

Correlation analysis between variables was conducted (Table II) to examine the relationship patterns in the present sample. The results revealed positive beliefs about rumination to be significantly positively correlated with negative beliefs about rumination (.32, $p<.01$ ). Subsequently, emotion- oriented coping was found to be significantly positively associated with positive beliefs about rumination $(.40, \mathrm{p}<.01)$ and negative beliefs about rumination $(.37, p<.01)$.

Another opening in literature that was attended to in the present study was the mediating role of emotion- oriented coping between the metacognitive beliefs. The aforementioned paths were tested by AMOS software. The direct effects between positive beliefs about rumination and negative beliefs about rumination $\left(\beta=.31, p<.01, \Delta R^{2}=.23\right)$, the indirect effects between positive and negative beliefs about rumination through emotion oriented coping $(\beta=.10$, $p<.05)$ showed mediating effects of emotion oriented coping. The Figure I shows the structural equation model for the model. The model fit indices reflected an adequacy of the range for all indices also shown in the Figure I.

The Table III shows the direct, indirect and total effects regression coefficients for the study variables. The independent variable should significantly correlate with the both mediator and dependent variable as a prerequisite to mediation analysis ${ }^{14}$. This has been observed in the present investigation and the change in $R^{2}$ was seen from .23 in direct effects to .18 after introducing the mediator.

TABLE I: DEMOGRAPHIC INFORMATION ( $n=514)$

\begin{tabular}{|l|l|c|c|c|c|c|}
\hline \multicolumn{1}{|c|}{ Variables } & Categories & Mean & SD & Range & F & $\%$ \\
\hline Age & & 32.56 & 6.96 & 37 & 499 & \\
\hline Gender & Male & & & & 384 & 76 \\
\hline & Female & & & & 130 & 24 \\
\hline Marital Status & Married & & & & 332 & 33 \\
\hline & Unmarried & & & & 170 & 65 \\
\hline Family System & Nuclear & & & & 114 & 22 \\
\hline & Joint & & & & 315 & 61 \\
\hline Organization Type & Govt. & & & & 214 & 42 \\
\hline & Semi- Govt, & & & & 63 & 12 \\
\hline & Private & & & & 225 & 43 \\
\hline Work Experience & & 9.41 & 6.30 & 34 & 477 & \\
\hline Work hours & & 10.29 & 8.19 & 61 & 455 & \\
\hline Salary & & $48,310.80$ & $31,237.44$ & & 402 & \\
\hline
\end{tabular}


Metacognitive Self-Regulation: The Mediating Role of Emotion

TABLE II: CORRELATION MATRIX FOR POSITIVE AND NEGATIVE BELIEFS ABOUT RUMINATION SCALES, AND EMOTION- FOCUSED COPING $(n=514)$

\begin{tabular}{|c|c|c|c|c|c|c|}
\hline & Subscales & I & II & III & No. of Items & Alpha Coefficient \\
\hline I & PBRS & - & - & - & 9 & .85 \\
\hline II & NBRS & $.32^{* *}$ & - & - & 9 & .88 \\
\hline III & EOC & $.40^{* *}$ & $.37^{* *}$ & - & 16 & .77 \\
\hline${ }^{*} \mathrm{p}<.05,{ }^{* *} \mathrm{p}<.01$ &
\end{tabular}

\section{FIGURE I:}

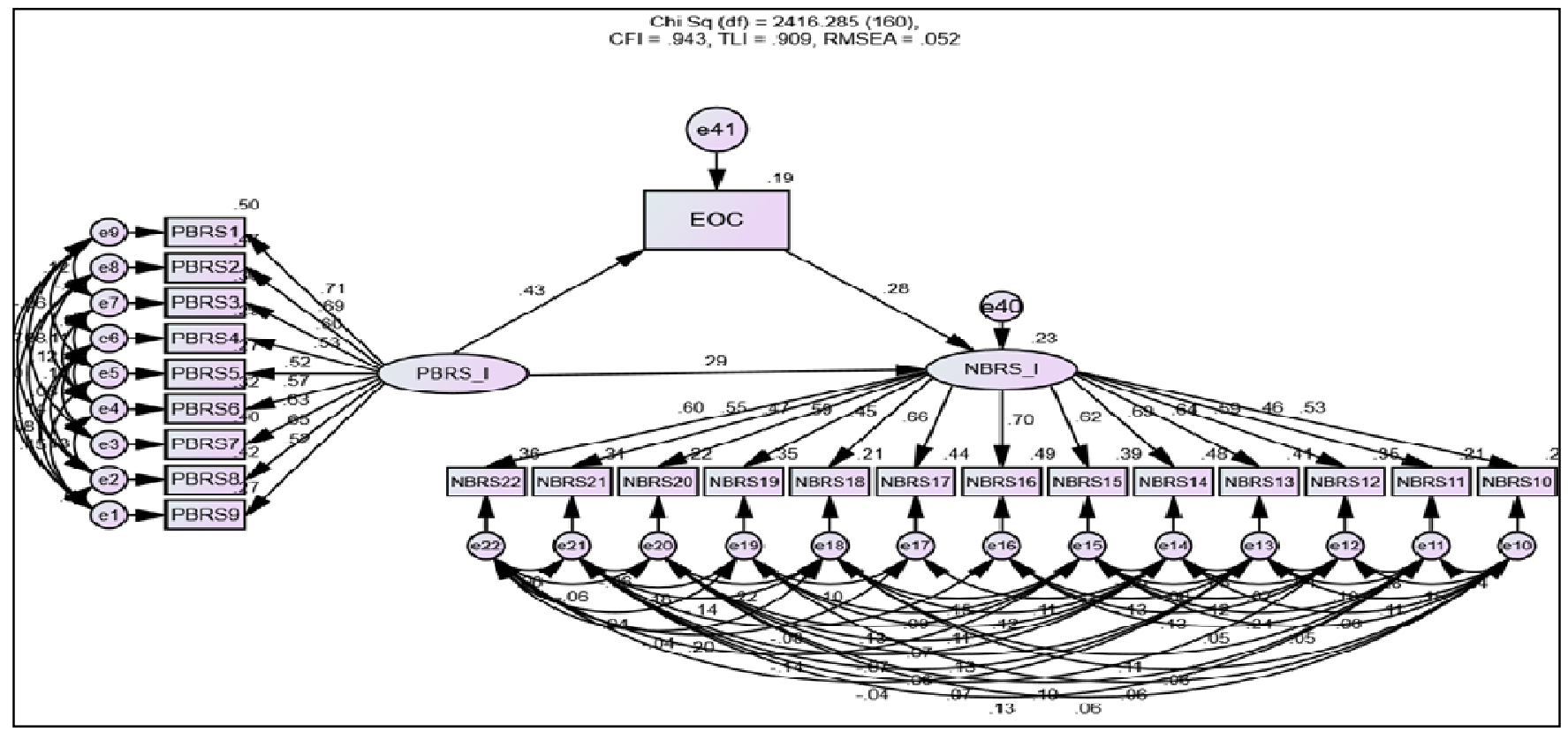

TABLE III: TABLE SHOWING DIRECT INDIRECT AND TOTAL EFFECTS ACROSS EMOTION ORIENTED COPING $(n=514)$

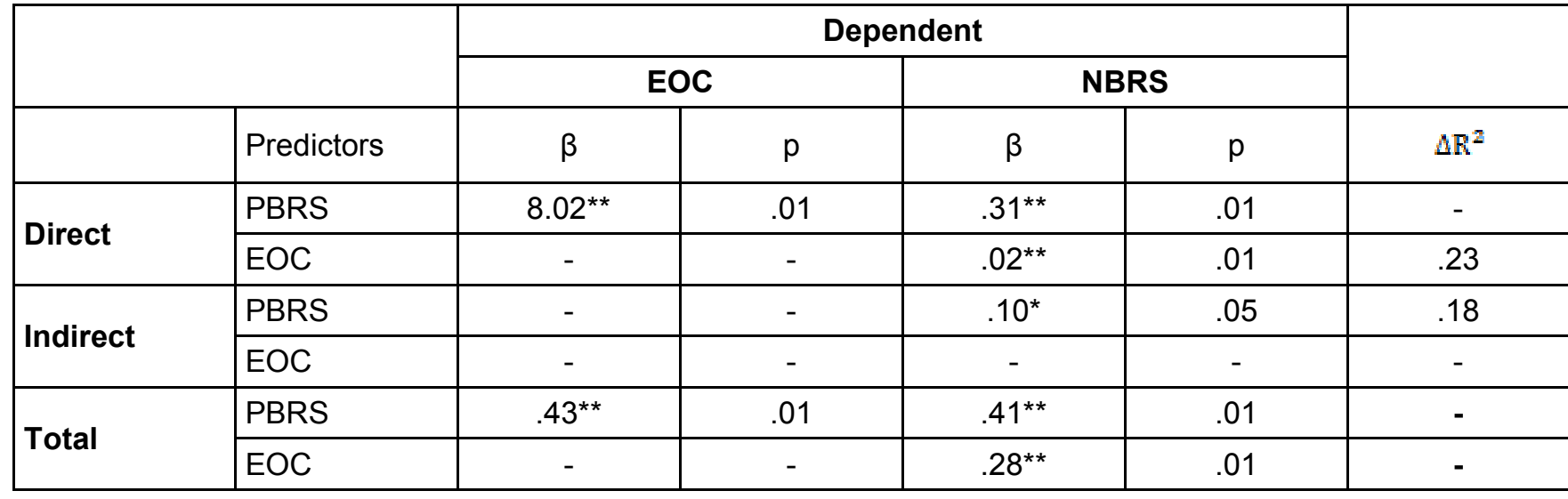

\section{DISCUSSION}

Literature has highlighted an association between positive and negative beliefs about rumination and emotional coping ${ }^{15}$. Some authors have indicated that these beliefs are the cause for the initiation and maintenance of emotional disorders ${ }^{16,1}$. The Self-Regulatory Executive Function model of rumination explains an extensive cognitive architecture for cyclic repetition (rumination) of thoughts that leads to more preservative thinking, enhances emotional negativity and is associated with anxiety ${ }^{17}$. The literature about the relationship between metacognitive beliefs and coping strategies are still scarce, although coping research is elaborate 
on other fronts of exploration ${ }^{18,19}$. The present research explored the relationship between metacognitive beliefs and emotion oriented coping.

The perspective of present study is indicative of a ruminative cognitive processing that is initiated in the face of challenges or stress ${ }^{20}$. Similar to previous studies $^{21}$, the results showed significantly positive relationships between PBRS and suggesting that elevation of either may contribute to the other. The previous literature submits PBRS and EOC to be positively correlated and this finding was corroborating in the present study disclosed by the correlations in similar directions. The mediating role of emotion oriented coping signifies that a parallel activation of positive and negative beliefs that has been previously associated with psychopathology can be intervened by an innate coping mechanism. This is further supported by the philosophy of Aristotle when he said "purging of the spirit of morbid and base ideas or emotions by witnessing the playing out of such emotions or ideas on stage" 22 .

\section{CONCLUSION}

Emotional coping is a type of catharsis that enables the discharge of certain strain factors thus facilitating a cognitive-emotional balance. Most of these associations have been implied and yet to be field tested, especially with respect to nonclinical population; the above relationships signpost towards the specification that beliefs underlie coping strategies. The positive relationship between positive beliefs about rumination, emotion oriented coping and negative beliefs about rumination specify that holding beliefs that rumination serves an advantage elevates the emotional content which is positively associated with an activation of the danger and uncontrollability of these thoughts.

\section{RECOMMENDATIONS}

Although the study attempted to include an elaborate sample yet some limitations must be highlighted. The cross sectional nature of the study confines concrete conclusions about the nature of the processing across time. Next, for future research attempts should include an exploration of individual differences which would further broaden the universality of the processing. It is thus recommended that the beliefs held for recurring thoughts should be explored before aiming at any cognitive intervention.

\section{Conflict of interest: None}

Funding: None

\section{REFERENCES}

1. Wells A, Matthews G. Attention and emotion: A Clinical Perspective. London: LEA. 1994.
2. Livingston, J. A. Metacognition: An Overview. 1997; Retrieved from http. www.gse.buffalo, edu/ fas/shuell/CEP564/Metaeog.htm.

3. Young EA, Nolen-Hoeksema S. Effect of ruminations on the saliva cortisol response to a social stressor. Psychoneuroendocrinology. 2001; 26(3):319-29.

4. Wells A, Matthews G. Modelling Cognition in Emotional Disorder: The S-REF model. Behavior Res Ther. 1996; 34(11-12):881-8.

5. Gross JJ, Thompson RA. Emotion Regulation: Conceptual Foundations. In J. J. Gross (Ed.), Handbook of emotion regulation (pp. 3-24). New York, NY, US: The Guilford Press. 2007.

6. Martin LL, Tesser A. Toward a motivational and structural theory of ruminative thought. In: J.S Ulemen \& J.A Bargh(Eds),Unintended thought (pp. 306-326). New York, NY, US: Guilford Press. 1989.

7. Martin LL, Tesser A. Some ruminative thoughts. In: R. S. Wyer Jr.(eds). Ruminative thoughts.(pp.1-47).Hillsdale, NJ: Erlbaum. 1996.

8. Gerard Methews Trevor A. Harley. Connectionist models of emotional distress and attentional bias. Cognition \& Emotion. 1996; 10(6):561-600. doi: $10.1080 / 026999396380060$

9. Wells A. Metacognitive therapy: Cognition applied to regulating cognition. Behav Cogn Psychother. 2008; 36(Special 6):651-8.

10. Ajzen, I. From Intentions to Actions: A Theory of Planned Behavior. In: J. Kuhl \& J. Beckman, eds. Action Control: From Cognition to Behavior. Heidelberg: Springer Berlin; 1985. p. 11-39.

11. Nolen-Hoeksema S, Parker LE, Larson J. Ruminative Coping with Depressed Mood following Loss. J Pers Soc Psychol. 1994; 67(1): 92-104.

12. Papageorgiou C, Wells A. Metacognitive beliefs about rumination in recurrent major depression. Cogn Behav Pract. 2001; 8(2):160-4.

13. Endler N, Parker J. State and trait anxiety, depression and coping styles. Aus $\mathrm{J}$ Psychol. 1990; 42(2):207-20.

14. Baron RM, Kenny DA. The Moderator-Mediator Variable Distinction in Social Psychological Research: Conceptual, Strategic, and Statistical Considerations. J Pers Soc Psychol. 1986; 51 (6):1173-82.

15. Papageorgiou C, Wells A. Nature, functions, and beliefs about depressive rumination. Depressive rumination. In: Papageorgiou C, Wells A, eds. Depresive Rumination: Nature, theory and treatment. John Wiley \& Sons Ltd. 2004. p.1-20.

16. Spada MM, Nikčević AV, Moneta GB, Wells A. Metacognition, perceived stress, and negative 
emotion. Personality and Individual Differences. 2008; 44(5):1172-81.

17. Nolen-Hoeksema S, Wisco BE, Lyubomirsky S. Rethinking rumination. Perspect Psychol Sci. 2008; 3(5):400-24. doi: 10.1111/j.1745-6924. 2008.00088.x.

18. Roelofs J, Huibers M, Peeters F, Arntz A, van Os $\mathrm{J}$. Rumination and worrying as possible mediators in the relation between neuroticism and symptoms of depression and anxiety in clinically depressed individuals. Behav Res Ther. 2008; 46(12):12839. doi: 10.1016/j.brat. 2008.10.002.

19. Skinner EA, Edge K, Altman J, Sherwood H. Searching for the structure of coping: a review and critique of category systems for classifying ways of coping. Psychol Bull. 2003; 129(2): 216269. doi: 10.1037/0033-2909.129.2.216.
20. Matthews, G, and Wells A. Rumination, depression, and metacognition: The S-REF model. In: Papageorgiou C, Wells A, eds. Depresive Rumination: Nature, theory and treatment. John Wiley \& Sons Ltd. 2004. p.125-151.

21. Garnefski N, Van Den Kommer T, Kraaij V, Teerds J, Legerstee J, Onstein E. The Relationship between Cognitive Emotion Regulation Strategies and Emotional Problems: Comparison between a Clinical and a Non Clinical Sample. Eur J Pers. 2002; 16(5):403-20.

22. Studtmann P. Aristotle Categories. Stanford Encyclopedia of Philosophy. 2007. Available from: https://plato.stanford.edu/entries/ aristotle categories/

AUTHOR AFFILIATION:
Aisha Muneer (Corresponding Author)
Ph.D. Scholar (Psychology)
National Institute of Psychology
Quaid-i-Azam University, Islamabad-Pakistan.
Email: aisham@nip.edu.pk,
$\quad$ aisha.kashif@comsats.edu.pk
Jamil A Malik
National Institute of Psychology
Quaid-i-Azam University, Islamabad-Pakistan.
Raiha Aftab
Lecturer, National Institute of Psychology
Quaid-i-Azam University, Islamabad-Pakistan.

